\title{
Modifications du comportement thermique et mécanique d'une serre induites par un brise-vent situé en amont
}

\author{
C Kittas \\ Technological Educational Institution of Messologi, Branch of Arta, Department of Crop Production, PO Box 110, 47100 Arta, Greece
}

(Reçu le 23 juillet 1992; accepté le 12 novembre 1992)

\begin{abstract}
Résumé - L'étude expérimentale de la déformation du profil de vitesse du vent autour d'une serre tunnel implantée à l'aval d'un brise-vent permet de mettre en évidence un fort ralentissement de l'écoulement (environ $30 \%$ de réduction de la vitesse moyenne) ainsi que des caractéristiques particulières de lignes de courant dues à la succession des 2 obstacles (formation d'une bulle d'air stationnaire entre le brise-vent et la serre). À partir de ces résultats expérimentaux, il est possible de déduire les conclusions suivantes:

- les pertes thermiques d'une serre protégée par un brise-vent sont diminuées d'environ 10-15\%;

- le réchauffement de l'air intérieur peut être supérieur à $1{ }^{\circ} \mathrm{C}$, fait qui implique une augmentation des besoins de ventilation;

- la structure de la serre protégée peut être allégée.
\end{abstract}

serre / brise-vent / écoulement d'air

Summary - Modifications in mechanical and thermal behaviour due to a windbreak in front of a greenhouse. A study of the wind velocity profile deformation around a semicylindrical greenhouse situated on the leeward side of a natural windbreak indicated that air flow showed a significant slowing down around the greenhouse $(\approx 30 \%$ reduction in mean wind velocity), and demonstrated particular streamline characteristics due to the succession of the 2 obstacles (formation of a stationary reversed air flow between the windbreak and the greenhouse).

From the above experimental results we have made the following conclusions: heat losses in a greenhouse protected by a windbreak are decreased by $\approx 10-15 \%$; overheating of greenhouse inner air can reach $>1{ }^{\circ} \mathrm{C}$, which implies an increase in greenhouse ventilation requirements; the structure of the protected greenhouse can be lighter.

greenhouse / windbreak / air flow

\section{INTRODUCTION}

Dans les régions soumises à des vents violents, fréquents et à direction dominante nettement marquée, les agriculteurs ont été amenés à créer des brise-vent afin de protéger leurs cultures.

C'est ainsi, par exemple, que dans le Midi de la France, où souffle le Mistral, qui est un vent de direction Nord, existent des réseaux de brise-vent qui délimitent des parcelles allongées d'orientation Est-Ouest. Dans la majorité des cas, les serres implantées dans de telles régions sont adossées aux brise-vent.
II nous a donc paru intéressant d'étudier les modifications du comportement aérodynamique et thermique d'une serre, consécutives à la présence du brise-vent afin de pouvoir ainsi mettre en évidence les avantages et les inconvénients de son utilisation.

\section{ÉCOULEMENT DE L'AIR AUTOUR D'UNE SERRE PROTÉGÉE PAR UN BRISE-VENT}

L'étude expérimentale a été effectuée au cours d'un séjour en France, à la station de bioclimatologie du centre de recherches agronomiques 
d'Avignon. Le détail des mesures et des calculs est présenté dans Kittas (1992). Cette étude a permis de représenter la déformation du profil des vitesses du vent au voisinage de la paroi d'une serre en plastique protégée du vent dominant par un brise-vent naturel mort constitué de cannes de Provence (Arundo donax) fichées dans le sol et présentant une porosité moyenne de $50 \%$.

On constate (fig 1) que :

a) I'obstacle que représente le brise-vent perturbe l'écoulement de l'air autour de la serre. La succession des 2 obstacles que constituent le brise-vent et la serre a comme résultat la formation d'une "bulle d'air" stationnaire, de faible vitesse, située entre les 2 obstacles. Une partie de l'écoulement se trouve ainsi bloquée en amont de la serre. Le reste de l'écoulement passe par dessus la serre avec un resserrement des lignes de courant et une inclinaison relativement faible, provoquant ainsi au voisinage de la paroi de serre, une accélération de la vitesse du vent, une chute des pressions et un appel d'air vers le haut par "effet Coanda» - effet qui traduit l'action de la force centrifuge agissant sur une particule d'air et qui est d'autant plus important que le rayon de courbure de la ligne de courant est plus faible (Plate, 1971) - moins importants que dans le cas de la serre sans le brise-vent où toute la veine incidente serait obligée de passer par dessus la serre. On peut donc conclure, que la présence du brise-vent diminue le champ des vitesses et des pressions autour de la serre.

b) la présence de la serre diminue la zone d'action du brise-vent. En effet, à partir de la zone faîtière, il se produit un décollement des filets d'air, qui, en état de dépression, vont se rabattre vers le sol à l'aval de la serre. C'est ainsi, par exemple, que le brise-vent sans la présence de la serre permet une réduction de la vitesse du vent mesurée à la hauteur $H / 4$ (où $H$ est la hauteur du brise-vent) de l'ordre de $50 \%$ à une distance de $12 \mathrm{H}$ et d'environ $25 \%$ à une distance de $18 H$ (Nägeli, 1953). La présence de la serre modifie ces distances à environ $4 \mathrm{H}$ et $6 \mathrm{H}$ respectivement.

\section{DISCUSSION}

On sait que la vitesse du vent intervient tant sur le niveau des échanges par convection au niveau des parois (Businger, 1966) que sur l'importance des fuites, contrôlées - ouvrants de ventilation statique (Baille, 1975) - ou non - défauts d'étanchéité (Gudehus, 1977) -. Une réduction de la vitesse du vent induite par la présence du brise-vent amène donc :

a) une diminution des échanges convectifs entre la paroi de la serre et son environnement;

b) une diminution des échanges d'air au niveau des ouvertures de la serre, dans la mesure où on suppose que le flux d'air à travers les ouvrants est proportionnel à la vitesse du vent en amont (Bot, 1983).

Dans ce qui suit, un essai de quantification de ces effets est présenté.

En admettant que les lignes de courant correspondant à une hauteur égale à la moitié de la hauteur du brise-vent représentent sensiblement l'écoulement moyen dans la zone dont la hauteur est égale à celle du brise-vent (Guyot, 1977), on peut déduire, à partir de la figure 1 , que le brise-

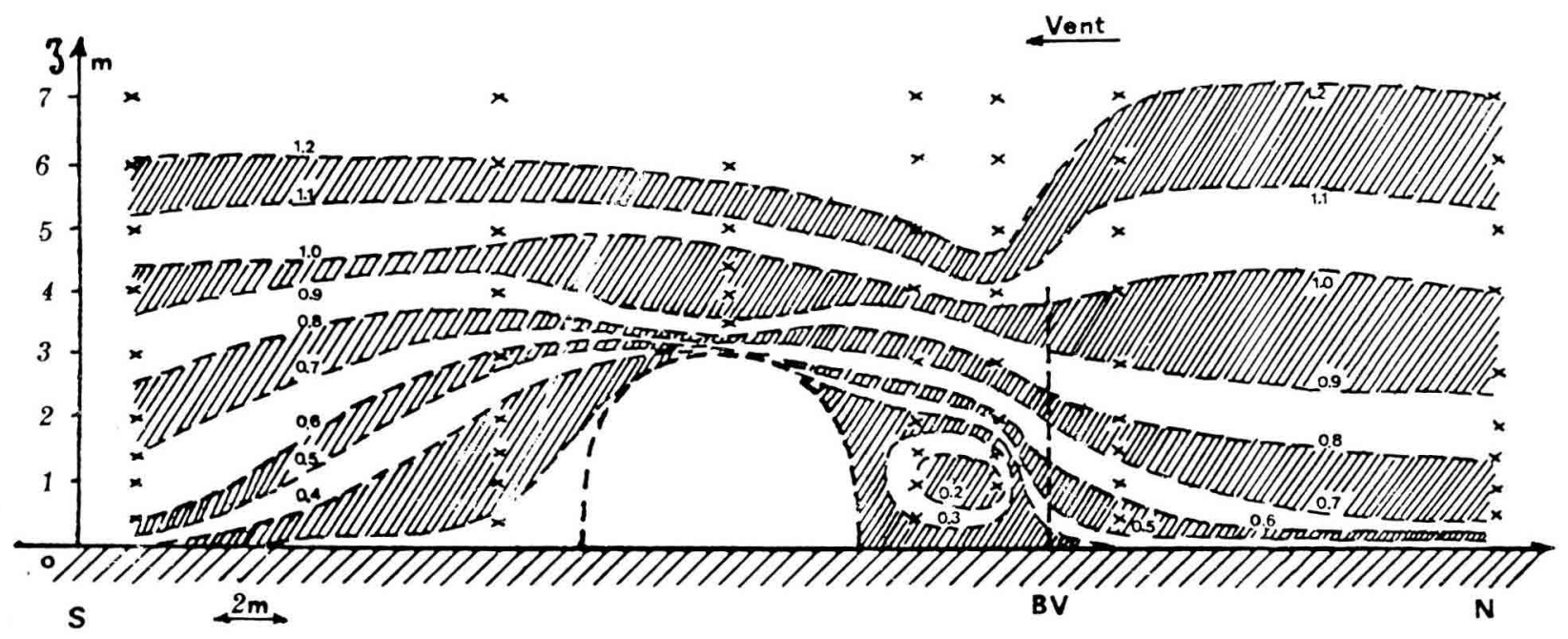

Fig 1. Vitesses relatives autour d'une serre implantée derrière un brise-vent (la vitesse de référence est mesurée en zone dégagée à une hauteur de $4 \mathrm{~m}$ ). 
vent entraîne une réduction de la vitesse du vent de l'ordre de $30 \%$ au niveau de référence de $H / 2$. En retenant l'expression proposée par Sheard (1978) selon laquelle les pertes de chaleur (Q) d'une serre plastique simple paroi s'expriment à partir de la relation

$$
Q=(4,76+0,52 \mu) \Delta T \quad\left(\text { en W. } \mathrm{m}^{-2}\right)
$$

où $\Delta T=$ différence de température de l'air entre extérieur et intérieur $\left(\mathrm{en}^{\circ} \mathrm{C}\right)$

et $\mu=$ vitesse du vent (en m. $\left.\mathrm{s}^{-1}\right)$

on constate que, pour une réduction de $30 \%$ de la vitesse du vent, les déperditions thermiques diminuent d'environ $10-15 \%$, pour une vitesse du vent extérieur de l'ordre de $5 \mathrm{~m} . \mathrm{s}^{-1}$.

D'autre part, en retenant l'expression proposée par Kittas (1987) selon laquelle, en régime diurne, l'amplitude des températures d'air entre l'intérieur et l'extérieur $(\Delta T)$ sous une serre fermée, cultivée et bien arrosée est donnée par :

$$
\Delta T=\frac{0,017 R G+2,62}{1+0,089 \mu^{0,8}} \quad\left(\text { en }^{\circ} \mathrm{C}\right)
$$

où $R G$ = rayonnement solaire global extérieur (en W. $\mathrm{m}^{-2}$ ).

On constate qu'une diminution de $30 \%$ de $\mu$ (pour des vitesses de l'ordre de $5 \mathrm{~m} . \mathrm{s}^{-1}$ et des valeurs de RG de l'ordre de $800 \mathrm{~W} \cdot \mathrm{m}^{-2}$ ) conduit à un réchauffement supérieur à $1{ }^{\circ} \mathrm{C}$, par rapport à une serre non protégée. Cette surchauffe implique donc une augmentation des besoins de ventilation, de l'ordre de $25 \%$ pour un $\Delta T$ de l'ordre de $5{ }^{\circ} \mathrm{C}$ (fig 2, d'après Kittas et al, 1987).

c) la figure 1 fait apparaître une autre «bulle d'air» entre les vitesses réduites 0,9 et 1,0 audessus du sommet de la serre. L'allure des lignes de courant permet de déduire que la présence de la serre perturbe l'écoulement derrière le brise-vent jusqu'à un niveau situé entre ces 2 lignes de courant. On peut donc en conclure que, pour une bonne protection, la hauteur du brisevent ne doit pas être inférieure à ce niveau qui, dans le cas de notre dispositif, correspond à 11,5 $\mathrm{m}$ au-dessus du faîtage de la serre. Sinon, compte tenu de la chute des lignes de courant au-dessus de la serre, les lignes correspondant à des vitesses supérieures «entreraient» dans la zone perturbée par la serre.

En admettant que les charges dues au vent $\left(W_{p}\right)$ qui s'exercent sur une surface de la cons-

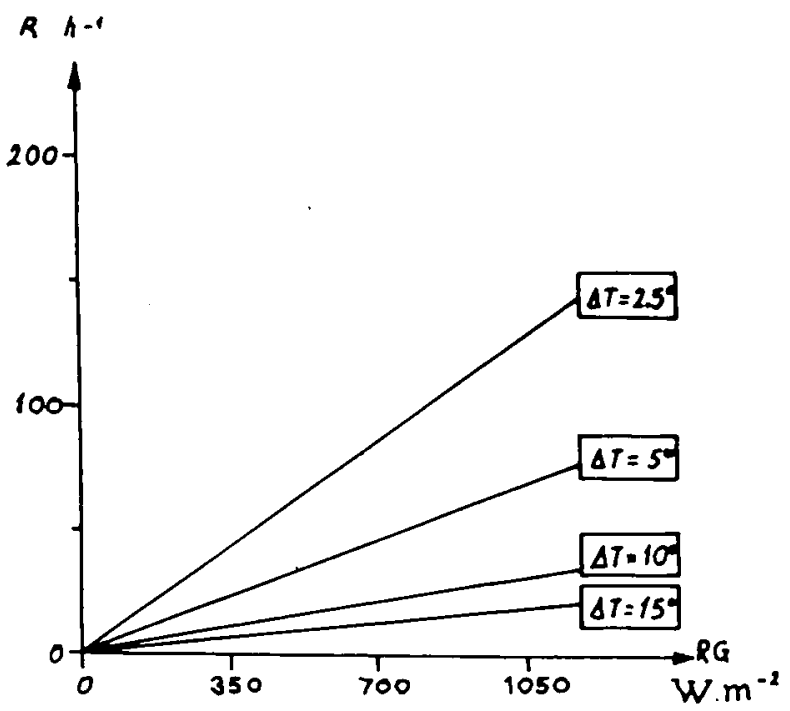

Fig 2. Estimation du taux horaire de renouvellement d'air ( $R$ ) pour limiter l'échauffement $(\Delta T)$ à l'intérieur d'une serre cultivée bien arrosée, en fonction du rayonnement solaire ( $R G$ ), d'après Kittas et al (1987).

truction, sont données par la relation (Anonyme, 1978) :

$$
W_{p}=C_{p} \cdot q \quad(\text { en } \mathrm{Pa})
$$

où

$C_{p}=$ coefficient de forme relatif à la surface $p$, qui dépend de la géométrie de la serre,

$q$ = pression dynamique qui se définit comme la pression exercée par le vent sur une surface placée perpendiculairement à sa direction (en $\mathrm{Pa}$ ) et sachant que la pression dynamique est proportionnelle à la vitesse du vent au carré, on s'aperçoit qu'une diminution de la vitesse du vent de $30 \%$, entraînée par le brise-vent, implique une diminution des $W_{p}$ de l'ordre de $50 \%$ et permet ainsi d'alléger significativement la construction.

\section{CONCLUSION}

Le brise-vent modifie considérablement le champ des vitesses autour d'une serre implantée à son aval. La serre se trouve placée dans un écoulement fortement freiné par rapport à l'écoulement qu'on aurait sans brise-vent. Ce ralentissement a des implications sur le comportement aérodynamique et énergétique de la serre. 
C'est ainsi que le brise-vent :

- permet d'alléger la construction dans la mesure où sont diminués les efforts dus au vent,

- permet d'obtenir en période de chauffage des économies d'énergie de l'ordre de $10 \%$ à $15 \%$ à condition que sa hauteur soit supérieure d'environ $1 \mathrm{~m}$ à la hauteur de la serre,

- augmente significativement les besoins de ventilation en période estivale.

On peut ainsi conclure que l'avantage de l'utilisation de brise-vent est net lorsqu'il s'agit de serres équipées de systèmes de chauffage et situées dans des zones froides et ventées. Par contre, dans le cas où les serres sont destinées à des cultures qui se prolongent durant la période estivale, la présence de brise-vent impliquera une aggravation des problèmes de ventilation et de refroidissement de l'atmosphère de la serre.

Par ailleurs, la présence de la serre diminue la longueur de la zone de protection du brise-vent et, par conséquent, la hauteur effective de ce dernier.

\section{RÉFÉRENCES}

Anonyme (1978) Serres, conditions de construction. Netherlands Normalisatie Institut (NL). No NEN 3859, $1^{\text {re }}$ éd, $20 \mathrm{p}$

Baille A (1975) Étude de l'influence du positionnement des aérations sur les champs de température et de vitesse à l'intérieur des tunnels de semi-forçage. Ann Agron 26, 265-275

Bot GPA (1983) Greenhouse climate: from Physical Processes to Dynamic Model. Ph D Thesis, Agricultural University Wageningen, Netherlands, $240 p$

Businger JA (1966) The glasshouse climate. In: Physics of plant environment (WR Van Wijk ed), North Holland Publ Company, Amsterdam

Gudehus H (1977) Determination of air exchanges figures with regard to closed greenhouses. Acta Hortic 76, 301-304

Guyot G (1977) Les effets aérodynamiques des brisevent. Prococlim, Tome 8E, No 3, 157-188

Kittas C (1987) Un modèle d'estimation des déperditions énergétiques diurnes des serres. Agronomie $7(8), 175-181$

Kittas C (1992) Influence d'un brise-vent sur les pertes convectives et radiatives d'une serre. Bound Layer Meteor (in press)

Kittas C, Chiapale JP, Villèle (de) O, Aries F (1987) Paramètres significatifs du déterminisme de la température de l'air de la serre. Agric For Meteorol 40, 265-277

Kozai T, Sase S (1978) A simulation of natural ventilation for multispan greenhouse. Acta Hortic 87, 3950

Nägeli W (1953) Untersuchungen über die Windverhältnisse in Bereich von Schilfrohwänden. Ann Inst Fédéral Rech For, Suisse 29 (2), 213-266

Plate EJ (1971) The aerodynamics of shelter belts. Agric For Meteorol 8(3), 203-222

Sheard GF (1978) Shelter and the effect of wind on the heat loss from greenhouse. Symp on more profitable use of energy in protected cultivation. Alnrap (Sweden) Aug ( $\mathrm{R}$ Larsen and $\mathrm{HH}$ Van der Borg, eds). Acta Hortic 76, 357-360 